\title{
Immunomodulatory Effects of Placenta-derived Mesenchymal Stem Cells on T Cells by Regulation of FoxP3 Expression
}

\author{
Soo-Hwan Kim ${ }^{1,2}$, Jieun Jung ${ }^{3}$, Kyung Jin Cho ${ }^{1,4}$, Jong-Ho Choi ${ }^{3}$, \\ Hyeong Seon Lee ${ }^{5}$ Gi Jin Kim ${ }^{3, *}$, Seung Gwan Lee ${ }^{1,4, *}$ \\ ${ }^{I}$ Department of Integrated Biomedical and Life Science, Graduate School, Korea University, Seoul, Korea \\ ${ }^{2}$ Department of Biomedical Laboratory Science, Gimcheon University, Gimcheon, Korea \\ ${ }^{3}$ Placenta Research Laboratory, Department of Biomedical Science, CHA University, Seongnam, Korea \\ ${ }^{4}$ Faculty of Health and Environmental Science, College of Health Science, Korea University, Seoul, Korea \\ ${ }^{5}$ Department of Biomedical Laboratory Science, fungwon University, Goesan, Korea
}

The immunomodulatory effects of mesenchymal stem cells (MSCs) are an important mediator of their therapeutic effects in stem cell therapy and regenerative medicine. The regulation mechanism of MSCs is orchestrated by several factors in both intrinsic and extrinsic events. Recent studies have shown that the dynamic expression of cytokines secreted from MSCs control T cell function and maturation by regulating the expression of FoxP3, which figures prominently in $\mathrm{T}$ cell differentiation. However, there is no evidence that placenta-derived mesenchymal stem cells (PD-MSCs) have strong immunomodulatory effects on $\mathrm{T}$ cell function and maturation via FoxP3 expression. Therefore, we compared the expression of FoxP3 in activated T cells isolated from peripheral blood and co-cultured with PD-MSCs or bone marrow-derived mesenchymal stem cells (BM-MSCs) and analyzed their effect on $\mathrm{T}$ cell proliferation and cytokine profiles. Additionally, we verified the immunomodulatory function of PD-MSCs by siRNA-mediated silencing of FoxP3. MSCs, including PD-MSCs and BM-MSCs, promoted differentiation of naive peripheral blood T cells into $\mathrm{CD} 4+\mathrm{CD} 25+\mathrm{FoxP} 3+$ regulatory $\mathrm{T}$ (Treg) cells. Intriguingly, the population of $\mathrm{CD} 4+\mathrm{CD} 25+\mathrm{FoxP} 3+$ Treg cells co-cultured with PD-MSCs was significantly expanded in comparison to those co-cultured with BM-MSCs or WI38 cells $(\mathrm{p}<0.05, \mathrm{p}<0.001)$. Dynamic expression patterns of several cytokines, including anti- and pro-inflammatory cytokines and members of the transforming growth factor-beta (TGF- $\beta$ ) family secreted from PD-MSCs according to FoxP3 expression were observed. The results suggest that PD-MSCs have an immunomodulatory effect on T cells by regulating FoxP3 expression.

Keywords: Placenta-derived mesenchymal stem cells, Immunomodulatory effects, Regulatory T cell, FoxP3, Cytokines

Received April 3, 2018, Revised July 23, 2018, Accepted July 31, 2018, Published online October 31, 2018

Correspondence to Seung Gwan Lee

Faculty of Health and Environmental Science, College of Health Science, Korea University, 145 Anam-ro, Sungbuk-gu, Seoul 02841, Korea

Tel: +82-2-940-2810, Fax: +82-2-917-2388, E-mail: seunggwan@korea.ac.kr

Co-Correspondence to Gi Jin Kim

Department of Biomedical Science, CHA University, 335 Pangyo-ro, Bundang-gu, Seongnam 13488, Korea

Tel: +82-31-881-7145, Fax: +82-31-881-4102, E-mail: gjkim@cha.ac.kr

${ }^{*}$ These authors contributed equally to this work.

(a) This is an open-access article distributed under the terms of the Creative Commons Attribution Non-Commercial License (http://creativecommons.org/licenses/by-nc/4.0/), which permits unrestricted non-commercial use, distribution, and reproduction in any medium, provided the original work is properly cited.

Copyright (c) 2018 by the Korean Society for Stem Cell Research 


\section{Introduction}

Mesenchymal stem cells (MSCs) from various tissues and organs show potential for self-renewal and differentiation into several lineages such as endoderm, mesoderm, and ectoderm although their capabilities are limited as compared to those of embryonic stem cells. Additionally, MSCs have immunomodulatory properties through human leukocyte antigen (HLA)-ABC ${ }^{\text {high }}$ and HLA-DR ${ }^{\text {low }}$ expression and the secretion of cytokines capable of regulating immune cell activity. This immunomodulation is mediated by cell-cell interactions between immune cells and MSCs as well as the soluble factors secreted from MSCs $(1,2)$. MSCs produce indoleamine 2,3-dioxygenase (IDO), and nitric oxide,which suppress the maturation and activation of dendritic cells and natural killer (NK) cells, differentiation of macrophages, and proliferation of $\mathrm{T}$ cells (3). The expression of pro-inflammatory cytokines, such as interferon-gamma (IFN- $\gamma$ ), interleukin (IL)-6, and prostaglandin E2, is regulated by MSCs when an inflammatory response is induced in vitro or in vivo (4). Hence, MSCs have the therapeutic potential to repair damaged tissues by regulating inflammation (5). Moreover, recent studies have demonstrated that MSC therapy in combination with solid organ transplantation is both effective and safe $(6,7)$.

Treg cells are characterized by high expression of Cluster of diffrentiation (CD)4, CD25, FoxP3, and IL-2 receptor alpha-chain. Treg cells are allegedly involved in the immunosuppressive effect of MSCs and can induce tolerance to self-antigens and regulation of the immune system. The population of Treg cells in humans comprises less than $3 \%$ of total CD4+ cells, yet they play a key role in immune homeostasis (8). Furthermore, Treg cells have the capacity to suppress the immune response in several inflammatory and autoimmune diseases, and upon organ transplantation (9). A recent study using in vivo imaging have highlighted that bone marrow-derived Treg cells may critically suppress the rejection of allo-hematopoietic stem cells in immunocompetent mouse models in an IL-10 dependent manner (10). The development and maintenance of Treg cells is highly dependent on co-stimulation by various cytokines, including IL-2, transforming growth factor-beta (TGF- $\beta$ ), and suppressor of cytokine signaling-1. In addition, Treg regulatory functions are mediated through cell-cell contact with the cell being suppressed $(11,12)$. Treg cells can be identified by their expression of the transcription factor forkhead box P3 (FoxP3) and the cytotoxic T lymphocyte antigen-4 (CTLA-4). Importantly, FoxP3 not only is an essential marker and regu- lator of Treg cells,it also modulates differentiation through genetically programming cell fate $(13,14)$. FoxP3 regulates the suppressive activity of Treg cells through three acetylation sites in the FoxP3 gene: K31, K262, and K267 (15).

Placenta-derived mesenchymal stem cells (PD-MSC), which are one early developmental source of several types of fetal tissue-derived mesenchymal stem cells, have shown therapeutic effects in several degenerative diseases $(16,17)$. Earlier studies have shown that PD-MSCs strongly suppress $\mathrm{T}$ lymphocyte proliferation through direct and indirect interactions owing to their high expression of immunomodulatory factors, including HLA-G, IDO, and various immunomodulatory cytokines (18-20). These findings are supported by observations that co-transplantation of PD-MSCs with cord blood in severe combined immunodeficient mice enhances the efficacy of engraftment without PD-MSC immunorejection $(21,22)$.

Previously, we demonstrated that chorionic plate-derived MSCs, one type of PD-MSCs, have HLA-G expression and cytokine secretion profiles different from those of bone marrow-derived mesenchymal stem cells (BM-MSCs) and adipose-derived MSCs, and moreover, they display higher potential for inhibition of $\mathrm{T}$ cell proliferation from an immunosuppressive point of view (23). However, no previous studies have assessed how PD-MSCs regulate Treg cell maturation and function in comparison to other MSCs. Thus, it is necessary to examine how the regulation of FoxP3 expression in the PD-MSC co-culture system functions as an immunosuppressive mechanism in the proliferation and maturation of $\mathrm{T}$ cells. Therefore, this study aimed to compare the expression pattern of FoxP3 in activated T cells co-cultured with either PD-MSCs or BM-MSCs. In conjunction, we evaluated whether down-regulation of FoxP3 expression by small interfering RNA (siRNA) treatment inhibited $\mathrm{T}$ cell proliferation or affected the cytokine profiles in our co-culturing system.

\section{Materials and Methods}

\section{Cell Culture}

BM-MSCs and normal fibroblast cells (WI38) were purchased from Cambrex Bioscience Walkersville (East Rutherford, NJ, USA) and ATCC (Manassas, VA, USA), respectively. Cells were cultured in alpha-MEM (Invitrogen, Carlsbad, CA, USA) containing 10\% fetal bovine serum (FBS), $1 \%$ penicillin/streptomycin $(\mathrm{P} / \mathrm{S}, 100 \mathrm{mg} / \mathrm{mL}$; Gibco-BRL, NY, USA), and $2 \mathrm{mM}$ L-glutamine (GibcoBRL). PD-MSCs were harvested from the inner side of the chorioamniotic membrane of the placenta as described 
previously (16). PD-MSCs and BM-MSCs at passages 6 to 8 were used for assays. The cells were treated with $50 \mu$ $\mathrm{g} / \mathrm{mL}$ of mitomycin C (MMC; Sigma-Aldrich) for $50 \mathrm{~min}$ to stop cell division and then used as feeder cells for co-culture with naïve or stimulated $\mathrm{T}$ cells isolated from peripheral blood (PB).

\section{T Cell Isolation and Activation Using Anti-CD3 and Anti-CD28}

To isolate CD4+ and CD25 + T cells, PB samples were obtained from three healthy donors $(\mathrm{N}=3)$, and $\mathrm{PB}$ mononuclear cells (PBMCs) were isolated by centrifugation using a Ficoll Hypaque (Histo-paque 1077; Sigma-Aldrich) density gradient as previously described (23). T cells were purified by exclusion of non-T cellsfrom the PBMCs by magnetic-activated cell sorting (MACS) using a Pan T Cell Isolation Kit II (Miltenyi Biotec, Auburn, CA, USA), and cultured in RPMI1640 medium containing 3\% FBS. For $\mathrm{T}$ cell stimulation, purified T cells $\left(5 \times 10^{5}\right)$ were cultured with $1.5 \mu \mathrm{g} / \mathrm{mL}$ soluble anti-CD3 (eBioscience, San Diego, CA, USA) and $1.5 \mu \mathrm{g} / \mathrm{mL}$ soluble anti-CD28 (eBioscience) for $72 \mathrm{~h}$. Successful stimulation (T cell clustering) was confirmed by bright-field microscopy.

\section{Co-culture of Activated T Cells with Feeder Cells}

To analyze the immunomodulatory effect of feeder cells, BM-MSCs, PD-MSCs, and WI38 cells were seeded at $2 \times 10^{3}, 1 \times 10^{4}$, and $2 \times 10^{4}$ cells per well, respectively, in a 96-well plate. After stabilization for $3 \mathrm{~h}, 5 \times 10^{5}$ of activated $\mathrm{T}$ cells were added and co-cultured with or without $1.5 \mu \mathrm{g} / \mathrm{mL}$ anti-CD3 and $1.5 \mu \mathrm{g} / \mathrm{mL}$ anti-CD28 monoclonal antibodies (mAbs) for $48 \mathrm{~h}$ at $37^{\circ} \mathrm{C}$ in $5 \% \mathrm{CO}_{2}$.

\section{Knock-down of FoxP3 Expression Using the AMAXA System}

For FoxP3-siRNA transfection of $\mathrm{T}$ cells, the 4D-Nucleofector ${ }^{\mathrm{TM}} \mathrm{X}$ kit (Lonza/Amaxa Biosystems, Walkersville, MD, USA) was used according to the manufacturer's instructions. FoxP3-siRNA and non-targeting siRNA (mock) were purchased from Ambion (Austin, TX, USA). The FoxP3-specific target sequences used in this study were 5'-GGGACCAAGAAGUGAGGUUTT-3' (sense) and 5'AACCUCACUUCUUGGUCCCTG-3' (antisense). After electronic transfection, the cells were activated with $1.5 \mu \mathrm{g} /$ $\mathrm{ml}$ of anti-CD3/anti-CD28 and co-cultured with $2 \times 10^{5}$ cells of MMC-exposed feeder cells (BM-MSCs, PD-MSCs, WI38).

\section{T Cell Proliferation Analysis}

To analyze the proliferative response during clonal ex- pansion of activated T cells in co-culture with feeder cells, we used the BrdU proliferation ELISA kit (Roche Diagnostics, Mannheim, Germany) according to the manufacturer's instructions. Colorimetric reactions were measured with an ELISA reader (Biotek, VT, USA) at $370 \mathrm{~nm}$. These experiments were performed in triplicate.

\section{RNA Isolation and Quantitative Reverse Transcriptase (qRT-)PCR Analysis}

To confirm FoxP3 gene expression, co-cultured feeder cells treated with MMC and activated T cells were harvested, homogenized and total RNA was isolated by Trizol reagent (Invitrogen) according to the manufacturer's instructions. The mRNA level of FoxP3 was determined by qRT-PCR using the SYBR ${ }^{\circledR}$ ExScript $^{\mathrm{TM}}$ RT-PCR Kit (TaKaRa, Tokyo, Japan) followed by detection on StepOne equipment (Applied Biosystems). The sequences of the FoxP3 primerpair were as follows: FoxP3 forward, 5'CACAACATGCGACCCCCTTTCACC-3'; FoxP3 reverse, 5'-AGGTTGT GGCGGATGGCGTTCTTC-3'. The target sequence was amplified by using the following thermal conditions: $2 \mathrm{~min}$ at $95^{\circ} \mathrm{C}$ and 40 cycles of $5 \mathrm{~s}$ at $95^{\circ} \mathrm{C}$ and $30 \mathrm{~s}$ at $59^{\circ} \mathrm{C}$. All reactions were carried out in triplicate.

\section{Fluorescence-activated Cell Sorting (FACS) Analysis}

To analyze intracellular FoxP3 expression, co-cultured feeder and activated $\mathrm{T}$ cells were dissociated in cell dissociation buffer (Gibco-BRL). The Treg detection kit (Miltenyi Biotec) was used according to the manufacturer's instructions. Stained cells were analyzed by multicolor flow cytometry on a FACS Calibur instrument (BD Biosciences, Mountain View, CA, USA) with CellQuest software (BD Biosciences).

\section{Bio-Plex Cytokine Assay}

Supernatants of $\mathrm{T}$ cells cultured in the absence or presence of feeder cells with or without $1.5 \mu \mathrm{g} / \mathrm{mL}$ anti-CD3/anti-CD28 mAbs were harvested after $72 \mathrm{~h}$ of cultivation. The Bio-Plex Pro Hu Cytokine assay 9-plex panel and Bio-Plex TGF- $\beta$ assay kit were used with the Bio-Plex Suspension Array System (Bio-Rad) to profile cytokine expression (IL-2, IL-4, IL-5, IL-6, IL-12, IL-13, IFN- $\gamma$, TGF- $\beta 1$, TGF- $\beta 2$, and TGF- $\beta 3$ ). The assay was performed according to the manufacturer's instructions. The concentrations of each cytokine were determined using an array reader. All samples were assayed in duplicate and analyzed with a Luminex 200 Labmap system (Luminex, Austin, TX, USA). Data analyses were performed using Bio-Plex Manager software version 4.1.1 
(Bio-Rad). Cytokine/chemokine concentrations were interpolated using an appropriate standard curve. All experiments were performed in triplicate.

\section{Statistical Analysis}

All experiments were replicated at least three times independently. The results are presented as the mean \pm standard deviation (SD). Group means were comparedusing Student's $t$-test or ANOVA followed by the LSD post-hoc test. Values of $\mathrm{p}<0.05$ were considered to indicate statistical significance.

\section{Results}

\section{Inhibition of PB T Cell Proliferation via Co-culture with MSCs}

Culturing of purified $\mathrm{T}$ cells in the presence of the $\mathrm{T}$ cell-activating mAbs anti-CD3/anti-CD28 for 3 days promoted the clustering of activated $\mathrm{T}$ cells. In contrast, $\mathrm{T}$ cell cluster formation was markedly reduced in co-culture with PD-MSCs or BM-MSCs (Fig. 1A). Furthermore, proliferation was significantly suppressed in $\mathrm{T}$ cells co-cultured with PD-MSCs and BM-MSCs when compared to cells in solo culture (Fig. 1B) or in co-culture with WI38 cells which were derived from human lung tissue (24) $\left({ }^{\#} \mathrm{p}<0.05\right.$, Fig. 1B) as indicated by BrdU ELISA (Fig. 1B). The proliferation-suppressive effect of PD-MSCs and BM-MSCs was dose-dependent. These data showed that PD-MSCs and BM-MSCs can inhibit PB $\mathrm{T}$ cell proliferation.

\section{MSCs Promote Differentiation of Naïve PB T Cells Into CD4+CD25+FoxP3 + Treg Cells}

Isolated PB $\mathrm{T}$ cells were co-cultured with PD-MSCs, BM-MSCs, and WI38 cells for 3 days in the presence of $\mathrm{mABs}$ anti-CD3/anti-CD28, and CD4+CD25+FoxP3+ $\mathrm{T}$ cells were identified by FACS. In co-culture-free conditions, the portion of CD4+CD25+FoxP3 $+\mathrm{T}$ cells was $3.54 \%$. In co-culture with PD-MSCs or BM-MSCs, CD4+ $\mathrm{CD} 25+\mathrm{FoxP} 3+\mathrm{T}$ cells increased to $10.89 \%$ and $6.37 \%$, respectively. However, in co-culture with WI38 cells, the portion of CD4 +CD25+FoxP3 + T cells was only 3.18\%, similar to that in co-culture-free conditions (Fig. 2A, upper panels). We found the highest rates of CD4+CD25+ FoxP3 + expression in T cells co-cultured with PD-MSCs, and these rates were significantly different from those in cells co-cultured with BM-MSCs $\left({ }_{\mathrm{p}}<0.05\right.$, Fig. $\left.2 \mathrm{~B}\right)$ and WI38 $\left({ }^{*} \mathrm{p}<0.001\right.$, Fig. 2B). In cells co-cultured with BM-MSCs, the fraction of CD4+CD25+FoxP3 + T cells was significantly higher than that in WI38 co-culture ${ }^{*} \mathrm{p}$
A
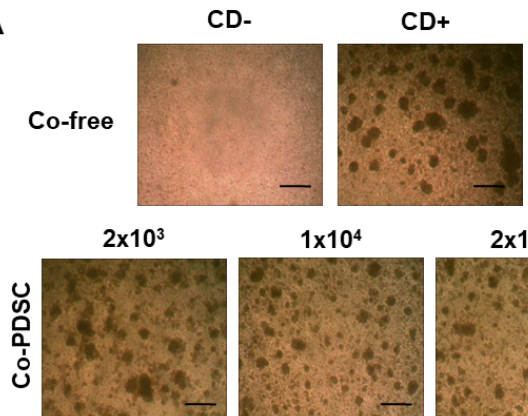

$1 \times 10^{4}$ $2 \times 10^{4}$
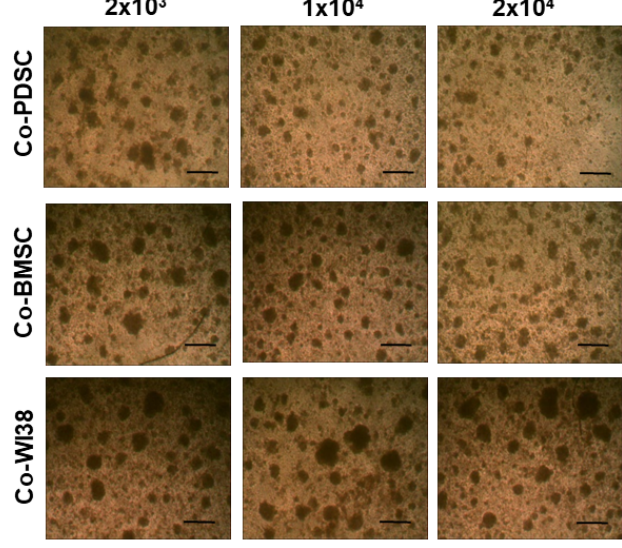

B

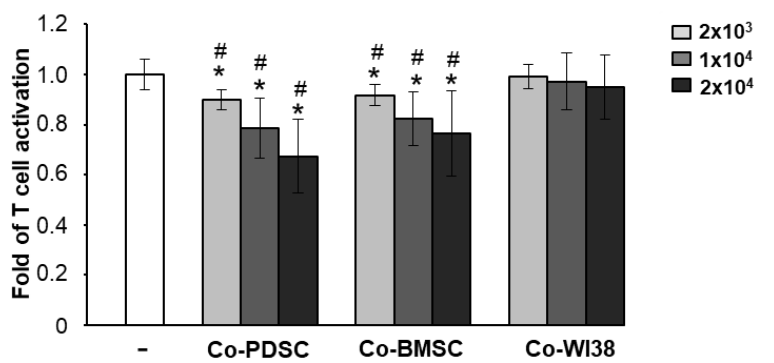

Fig. 1. Immunomodulatory effects of MSCs derived from placenta (PD-MSCs) and bone marrow (BM-MSCs) on activated PB T cells depend on co-cultured MSCs in a dose-dependent manner. (A) The clustering of activated $\mathrm{T}$ cells decreased with an increasing number of co-cultured MSCs, but not in co-culture with normal fibroblast WI38 cells. Scale bar $=50 \mu \mathrm{m}$. (B) Proliferation of T cells co-cultured with each of type of MSCs as determined by BrdU ELISA analysis after $72 \mathrm{~h}$ of culture. ${ }^{*} \mathrm{p}<0.05$ vs. co-culture-free cells. ${ }^{\#} \mathrm{p}<0.001$ vs. co-culture with WI38 cells.

$<0.001$, Fig. 2B).

To investigate the role of FoxP3 in Treg differentiation, we knocked down FoxP3 expression in purified T cells using siRNA. Mock siRNA was used as a control. FoxP3 mRNA levels were significantly decreased in siFoxP3treated $\mathrm{T}$ cells in all PD-MSC, BM-MSC, and WI38 co-culture conditions as compared to the respective siRNA mock controls ( ${ }^{*} \mathrm{p}<0.05$, Fig. 3). However, FoxP3 mRNA levels in $\mathrm{T}$ cells co-cultured with PD-MSCs were higher than those in co-culture with BM-MSCs $\left({ }^{\dagger} p<0.05\right)$ or WI38 cells $\left({ }_{\mathrm{p}}<0.05\right)$. Knockdown of FoxP3 significantly reduced Treg differentiation in both PD-MSC and BM-MSC co-cultures to the co-culture-free control level, 
A

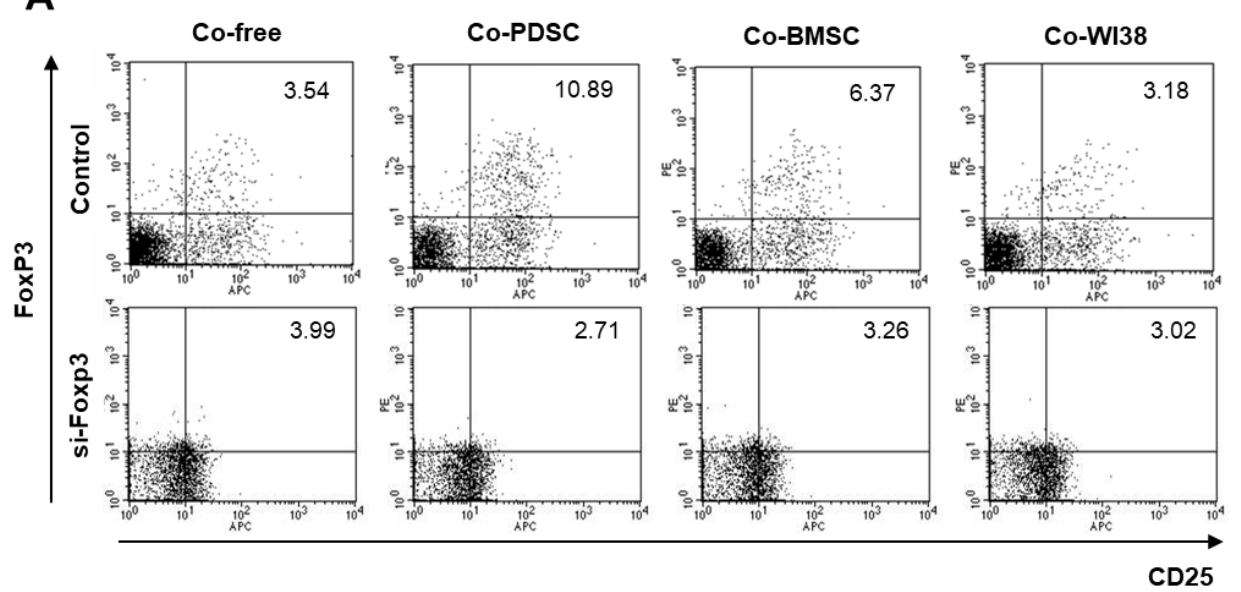

B

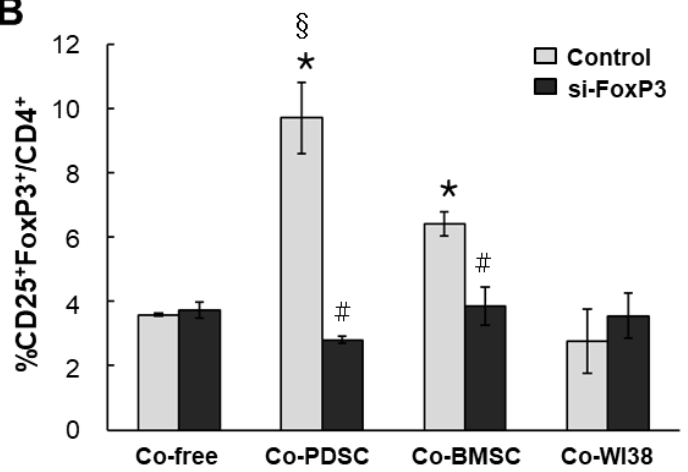

Fig. 2. Differentiation of naïve $\mathrm{PB} T$ cells into $\mathrm{CD} 4+\mathrm{CD} 25+$ FoxP3+ Treg cells when co-cultured with PD-MSCs, BM-MSCs, and WI38 cells. Dot plots of populations are shown in (A), and the bar graph shows the quantification of the FACS analysis data (B). ${ }^{*} \mathrm{p}<0.001$ vs. co-culture with WI38 cells. ${ }^{\#} \mathrm{p}<0.01$ vs. non-siFoxP3-treated cells. ${ }^{8} \mathrm{p}<$ 0.05 vs. co-culture with BMSCs.

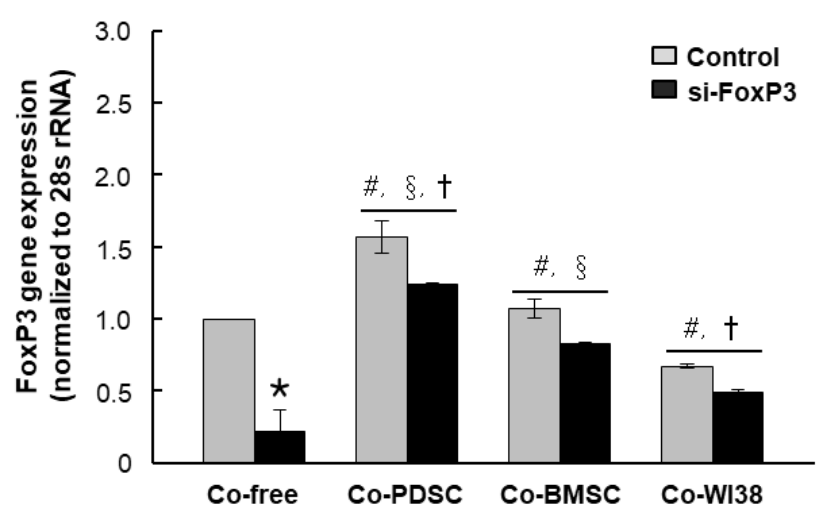

Fig. 3. FoxP3 gene expression in cells treated with FoxP3 and mock siRNA and co-cultured with PD-MSCs, BM-MSCs, and WI38 cells. FoxP3 gene expression was assessed by qRT-PCR (A). ${ }^{*} \mathrm{p}<0.05$ vs. mock control cells. ${ }^{\#} p<0.05$ vs. co-culture-free cells. ${ }^{\S} p<0.05$ vs. co-culture with WI38 cells. ${ }^{\dagger} p<0.05$ vs. co-culture with BM-MSCs.

with values of $2.71 \%$ and $3.28 \%$, respectively (Fig. 2A, lower panels, $\mathrm{p}<0.01$, Fig. 2B). These results indicated that MSCs promote differentiation of naïve $\mathrm{PB} T$ cells into $\mathrm{CD} 4+\mathrm{CD} 25+\mathrm{FoxP} 3+$ Treg cells, likely via FoxP3, and PD-MSCs are more effective regulators of $\mathrm{T}$ cell dif- ferentiation than BM-MSCs. However, when the si-FoxP3 group compared dot plots with each co-culture-free control group, overall protein expression of CD25 and FoxP3 was slightly elevated. The same results were obtained in repeated experiments several times, and it is necessary to investigate the cause in follow-up studies.

\section{FoxP3 Exerts Its Immunomodulatory Effects on T Cells Through Cytokine Regulation}

To investigate FoxP3 immunomodulatory effects on $\mathrm{T}$ cells further, we measured the levels of immune-regulatory cytokines secreted by siFoxP3- and mock-treated $\mathrm{T}$ cells in the absence or presence of feeder cells using multi-cytokine analysis. In Fig. 4, results for pro-inflammatory cytokines are shown in panel $\mathrm{A}$, and those for anti-inflammatory cytokines are presented in panel B. IL-2 and IL-12 levels were significantly lower in PD-MSC, BM-MSC, and WI38 co-cultures than in co-culture-free conditions. In co-culture-free conditions, IL-2 levels in siFoxP3-treated cell cultures were significantly decreased. IL-6 secretion was significantly higher in PD-MSC, BM-MSC, and WI38 co-culture conditions than in co-culture-free controls. In contrast, IL-6 secretion in 


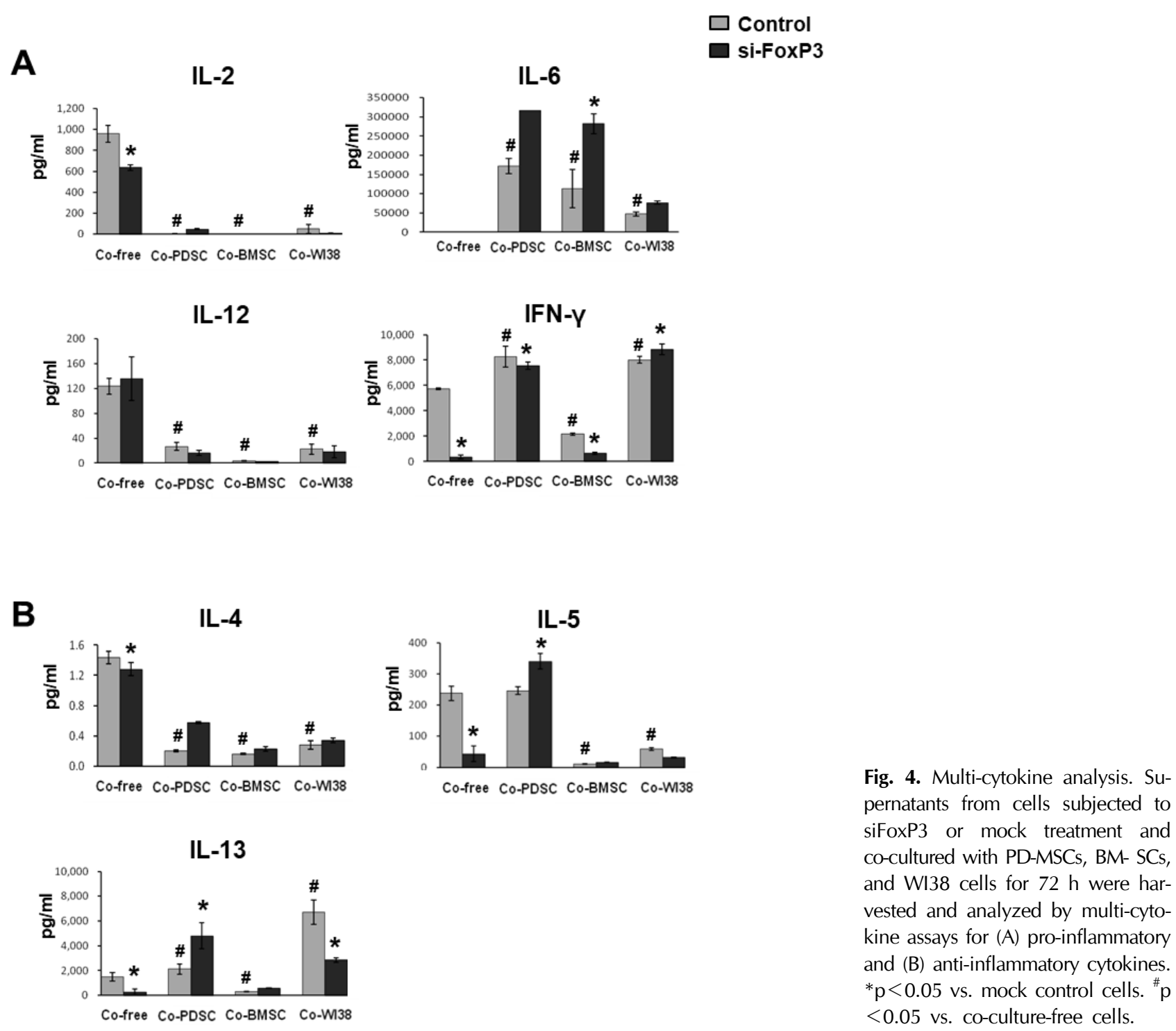

siFoxP3-treated cell cultures was significantly increased in comparison to siRNA mock control cells co-cultured with PD-MSCs or BM-MSCs $(\mathrm{p}<0.05)$. IFN- $\gamma$ levels were significantly higher in PD-MSC and WI38 co-culture conditions but lower in BMSC co-cultures than in the co-culture-free control and were significantly decreased in co-culture-free and PD-MSC or BM-MSC co-culture by siFoxP3-treatment $(\mathrm{p}<0.05)$.

As for the anti-inflammatory cytokines, IL-4 secretion was significantly lower in PD-MSC, BM-MSC, and WI38 co-cultures than in co-culture-free conditions $(\mathrm{p}<0.05)$. IL-5 levels were significantly lower in BM-MSC and WI38 $(\mathrm{p}<0.05)$, but not in PD-MSC co-cultures, than in co-culture-free condition. siFoxP3 treatment significantly lowered IL-5 secretion in co-culture-free condition, while it significantly induced IL-5 secretion in PD-MSC co-culture when compared to the respective mock controls ( $\mathrm{p}$ $<0.05$ ). IL-13 levels were significantly higher in PD-MSC and WI38 $(\mathrm{p}<0.05)$ but significantly lower in BM-MSC co-cultures than in co-culture-free condition. In siFoxP3-treated cells, IL-13 levels were significantly decreased in co-culture-free conditions and BM-MSC co-cultures; however, they were significantly increased in PD-MSC co-culture $(\mathrm{p}<0.05$, Fig. 4B).

The TGF- $\beta$ family is involved in immunmodulation as well as T-cell maturation (25). In Figure 5, the secretion patterns of the TGF- $\beta$ family members investigated, TGF- $\beta 1$, TGF- $\beta 2$, and TGF- $\beta$ 3, were similar. TGF- $\beta$ levels were significantly higher in PD-MSC and BM-MSC co-cultures than in co-culture-free and WI38 co-culture 

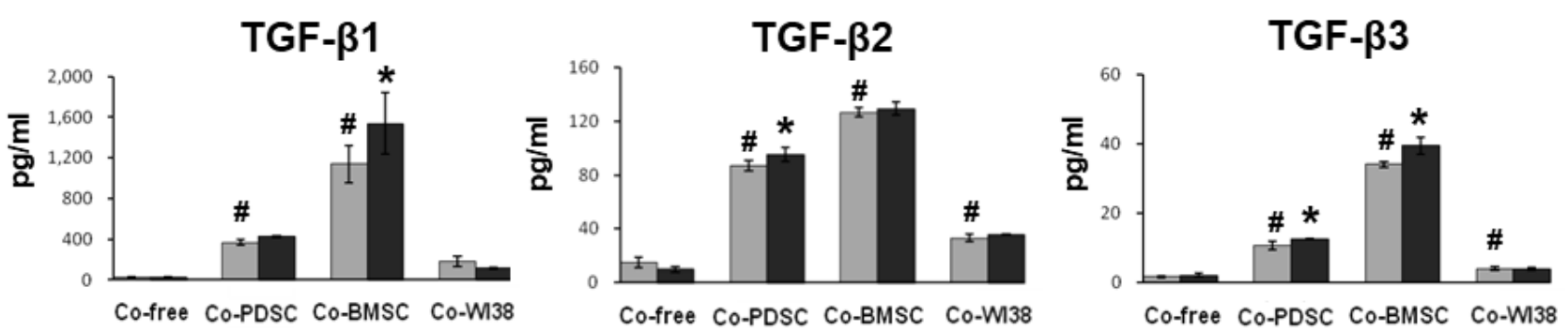

Fig. 5. Expression of TGF- $\beta$ family members in supernatants from cells subjected to siFoxP3 or mock treatment and co-cultured with PD-MSCs, BM-MSCs, and WI38 cells for $72 \mathrm{~h}$ were harvested and analyzed by multi-cytokine assays. ${ }^{*} \mathrm{p}<0.05$ vs. mock control cells. ${ }^{*} \mathrm{p}<0.05$ vs. co-culture-free cells.

conditions, for both siFoxP3-treated and mock controls ( $p$ $<0.05)$. In cultures of siFoxP3-treated cells, TGF- $\beta 2$ and TGF- $\beta 3$ levels were significantly increased in PD-MSC co-culture, and the TGF- $\beta 2$ level was significantly increased in BM-MSC co-culture $(\mathrm{p}<0.05)$. Together, these results indicated that MSCs can regulate the levels of antiand pro-inflammatory cytokines and TGF- $\beta$ family members, and FoxP3 might be a key factor in the immunomodulation of Treg cells by MSCs.

\section{Discussion}

Treg cells, which are required to maintain immunological tolerance, can be induced by several trophic factors secreted by MSCs such as TGF- $\beta$, IFN- $\gamma$, and IL-2 (26). The generation and development of natural Treg cells are affected by Treg-associated molecules including CD25, CTLA-4, IL-2, and FoxP3. Among these, FoxP3 is involved in Treg cell maturation and is stably expressed in mature Treg cells. High FoxP3 expression suppresses effector $\mathrm{T}$ cells directly or indirectly through activated antigen-presenting cells, resulting in immunological tolerance to self and non-self (27). Although no studies have compared FoxP3 expression patterns in Treg cells induced by MSCs isolated from different organs or tissues, recently, Jones et al. reported that placenta-derived MSCs can suppress allogeneic $\mathrm{T}$ cell proliferation through induction of IDO by IFN- $\gamma$ (19). In addition, Erkers et al. reported that the suppressive effect of placenta-derived MSCs, including decidual stromal cells isolated from the fetal membrane of term placenta, required cell-cell contact, which was consistent with an increased frequency of FoxP3+ Treg cells through IDO expression (28).
In the present study, we compared the expression of FoxP3 in activated $\mathrm{T}$ cells co-cultured with either PD-MSCs or BM-MSCs and analyzed the inhibition of T cell proliferation and differences in cytokine profiles. Moreover, we verified the immunomodulatory function of PD-MSCs using FoxP3 siRNA.

It has been recently reported that the differential secretome of stem cells is important for understanding the mechanisms underlying diverse therapeutic effects of a certain type of stem cells in several diseases (29-31). In support of this, in the present study, we found dynamically altered secretion of several cytokines from $\mathrm{T}$ cells co-cultured with PD-MSCs, BM-MSCs, or WI38 normal fibroblasts. Moreover, down-regulation of FoxP3 by siRNA decreased the secretion of several cytokines (e.g., IL-2, IFN- $\gamma$ ) that affect immune suppression.

It has been previously shown that in Treg cells, FoxP3 acts as a positive regulator of IL-2, and in turn, the IL-2-mediated JAK-STAT5 signaling pathway regulates FoxP3 expression in both mice and humans (32). These findings are in good accord with our observation that IL-2 expression in activated $\mathrm{T}$ cells was significantly decreased after siRNA-FoxP3 treatment. In addition, the expression of IL-6 -an important inflammatory cytokine-in MSCs, including PD-MSC and BM-MSC co-cultures, was significantly increased in siRNA-FoxP3-treated MSCs.

By profiling anti-inflammatory cytokines, we showed that the secretion of IL-4, IL-5, and IL-13 by T cells was increased by FoxP3 knockdown in co-cultures with MSCs but not WI38 cells, and the levels of these cytokines were higher in PD-MSC than in BM-MSC co-cultures. Thus, secretion of anti-inflammatory cytokines by $\mathrm{T}$ cells co-cultured with PD-MSCs was higher than that in co-culture 
with BM-MSCs, even when FoxP3 was down-regulated, implying that PD-MSCs have superior immunomodulatory capacity. Recent studies have found that BMSCs modulate immune responses through the expression of TGF- $\beta$ (25). These findings strongly correlate with the high secretion of TGF- $\beta 1,-\beta 2$, and $-\beta 3$ in BM-MSC co-cultures observed in this study. However, we found no difference in the expression of TGF- $\beta 2$ between the BM-MSC and PD-MSC co-cultures, regardless of FoxP3 expression.

In conclusion, our study indicated that PD-MSC could regulate Foxp3 expression in Treg by several inflammatory and anti-inflammatory cytokines. However, cytokine mechanism between PD-MSC and Foxp3 was not fully explained through this study. Furthermore, Future studies should focus on the mechanism of signal transduction between Foxp3 and each of the inflammatory cytokines regulated by PD-MSC or BM-MSC co-culture.

\section{Acknowledgments}

This work was supported by the Korea Healthcare Technology R\&D Project through the Korea Health Industry Development Institute (KHIDI), funded by the Ministry of Health \& Welfare, Republic of Korea (grant number : HI16C1559).

\section{Potential Conflict of Interest}

The authors indicate no potential conflicts of interest.

\section{References}

1. Nauta AJ, Fibbe WE. Immunomodulatory properties of mesenchymal stromal cells. Blood 2007;110:3499-3506

2. Quaedackers ME, Baan CC, Weimar W, Hoogduijn MJ. Cell contact interaction between adipose-derived stromal cells and allo-activated T lymphocytes. Eur J Immunol 2009;39:3436-3446

3. Engela AU, Baan CC, Dor FJ, Weimar W, Hoogduijn MJ. On the interactions between mesenchymal stem cells and regulatory $\mathrm{T}$ cells for immunomodulation in transplantation. Front Immunol 2012;3:126

4. Prasanna SJ, Gopalakrishnan D, Shankar SR, Vasandan AB. Pro-inflammatory cytokines, IFNgamma and TNFalpha, influence immune properties of human bone marrow and Wharton jelly mesenchymal stem cells differentially. PLoS One 2010;5:e9016

5. Hoogduijn MJ, Popp F, Verbeek R, Masoodi M, Nicolaou A, Baan C, Dahlke MH. The immunomodulatory properties of mesenchymal stem cells and their use for immunotherapy. Int Immunopharmacol 2010;10:1496-1500

6. Hoogduijn MJ, Popp FC, Grohnert A, Crop MJ, van Rhijn M, Rowshani AT, Eggenhofer E, Renner P, Reinders ME,
Rabelink TJ, van der Laan LJ, Dor FJ, Ijzermans JN, Genever PG, Lange C, Durrbach A, Houtgraaf JH, Christ B, Seifert M, Shagidulin M, Donckier V, Deans R, Ringden O, Perico N, Remuzzi G, Bartholomew A, Schlitt HJ, Weimar W, Baan CC, Dahlke MH. Advancement of mesenchymal stem cell therapy in solid organ transplantation (MISOT). Transplantation 2010;90:124-126

7. Tolar J, Villeneuve P, Keating A. Mesenchymal stromal cells for graft-versus-host disease. Hum Gene Ther 2011;22: 57-262

8. Feuerer M, Hill JA, Mathis D, Benoist C. Foxp3+ regulatory T cells: differentiation, specification, subphenotypes. Nat Immunol 2009;10:689-695

9. Wood KJ, Sakaguchi S. Regulatory $\mathrm{T}$ cells in transplantation tolerance. Nat Rev Immunol 2003;3:199-210

10. Fujisaki J, Wu J, Carlson AL, Silberstein L, Putheti P, Larocca R, Gao W, Saito TI, Lo Celso C, Tsuyuzaki H, Sato T, Côté D, Sykes M, Strom TB, Scadden DT, Lin CP. In vivo imaging of Treg cells providing immune privilege to the haematopoietic stem-cell niche. Nature 2011;474:21619

11. Sharma R, Sharma PR, Kim YC, Leitinger N, Lee JK, Fu SM, Ju ST. IL-2-controlled expression of multiple T cell trafficking genes and Th2 cytokines in the regulatory $\mathrm{T}$ cell-deficient scurfy mice: implication to multiorgan inflammation and control of skin and lung inflammation. J Immunol 2011;186:1268-1278

12. Takahashi R, Nishimoto S, Muto G, Sekiya T, Tamiya T, Kimura A, Morita R, Asakawa M, Chinen T, Yoshimura A. SOCS1 is essential for regulatory $\mathrm{T}$ cell functions by preventing loss of Foxp3 expression as well as IFN\{gamma and IL-17A production. J Exp Med 2011;208: 2055-2067

13. Fontenot JD, Gavin MA, Rudensky AY. Foxp3 programs the development and function of CD4+CD25+ regulatory T cells. Nat Immunol 2003;4:330-336

14. Hori S, Nomura T, Sakaguchi S. Control of regulatory T cell development by the transcription factor Foxp3. Science 2003;299:1057-1061

15. Kwon HS, Lim HW, Wu J, Schnölzer M, Verdin E, Ott M. Three novel acetylation sites in the Foxp3 transcription factor regulate the suppressive activity of regulatory $\mathrm{T}$ cells. J Immunol 2012;188:2712-2721

16. Lee MJ, Jung J, Na KH, Moon JS, Lee HJ, Kim JH, Kim GI, Kwon SW, Hwang SG, Kim GJ. Anti-fibrotic effect of chorionic plate-derived mesenchymal stem cells isolated from human placenta in a rat model of $\mathrm{CCl}(4)$-injured liver: potential application to the treatment of hepatic diseases. J Cell Biochem 2010;111:1453-1463

17. Parolini O, Caruso M. Review: Preclinical studies on placenta-derived cells and amniotic membrane: an update. Placenta 2011;32 Suppl 2:S186-S195

18. Ivanova-Todorova E, Mourdjeva M, Kyurkchiev D, Bochev I, Stoyanova E, Dimitrov R, Timeva T, Yunakova $M$, Bukarev D, Shterev A, Tivchev P, Kyurkchiev S. HLA-G expression is up-regulated by progesterone in mesenchymal 
stem cells. Am J Reprod Immunol 2009;62:25-33

19. Jones BJ, Brooke G, Atkinson K, McTaggart SJ. Immunosuppression by placental indoleamine 2,3-dioxygenase: a role for mesenchymal stem cells. Placenta 2007;28:1174-1181

20. Li C, Zhang W, Jiang X, Mao N. Human-placenta-derived mesenchymal stem cells inhibit proliferation and function of allogeneic immune cells. Cell Tissue Res 2007;330:437446

21. Hiwase SD, Dyson PG, To LB, Lewis ID. Cotransplantation of placental mesenchymal stromal cells enhances single and double cord blood engraftment in nonobese diabetic/severe combined immune deficient mice. Stem Cells 2009;27:2293-2300

22. Shi M, Liu ZW, Wang FS. Immunomodulatory properties and therapeutic application of mesenchymal stem cells. Clin Exp Immunol 2011;164:1-8

23. Lee JM, Jung J, Lee HJ, Jeong SJ, Cho KJ, Hwang SG, Kim GJ. Comparison of immunomodulatory effects of placenta mesenchymal stem cells with bone marrow and adipose mesenchymal stem cells. Int Immunopharmacol 2012;13: 219-224

24. Legrain Y, Touat-Hamici Z, Chavatte L. Interplay between selenium levels, selenoprotein expression, and replicative senescence in WI-38 human fibroblasts. J Biol Chem 2014; 289:6299-6310.

25. Svobodova E, Krulova M, Zajicova A, Pokorna K, Prochazkova J, Trosan P, Holan V. The role of mouse mesenchymal stem cells in differentiation of naive T-cells into anti-inflammatory regulatory $\mathrm{T}$-cell or proinflammatory helper T-cell 17 population. Stem Cells Dev 2012;21:901910

26. Caplan AI, Dennis JE. Mesenchymal stem cells as trophic mediators. J Cell Biochem 2006;98:1076-1084

27. Sakaguchi S. Naturally arising Foxp3-expressing CD25+ $\mathrm{CD} 4+$ regulatory $\mathrm{T}$ cells in immunological tolerance to self and non-self. Nat Immunol 2005;6:345-352

28. Erkers T, Nava S, Yosef J, Ringdén O, Kaipe H. Decidual stromal cells promote regulatory $\mathrm{T}$ cells and suppress alloreactivity in a cell contact-dependent manner. Stem Cells Dev 2013;22:2596-2605

29. Salgado AJ, Reis RL, Sousa NJ, Gimble JM. Adipose tissue derived stem cells secretome: soluble factors and their roles in regenerative medicine. Curr Stem Cell Res Ther 2010; 5:103-110

30. van Koppen A, Joles JA, van Balkom BW, Lim SK, de Kleijn D, Giles RH, Verhaar MC. Human embryonic mesenchymal stem cell-derived conditioned medium rescues kidney function in rats with established chronic kidney disease. PLoS One 2012;7:e38746

31. Wang Y, Bakota E, Chang BH, Entman M, Hartgerink JD, Danesh FR. Peptide nanofibers preconditioned with stem cell secretome are renoprotective. J Am Soc Nephrol 2011; 22:704-717

32. Murawski MR, Litherland SA, Clare-Salzler MJ, DavoodiSemiromi A. Upregulation of Foxp3 expression in mouse and human Treg is IL-2/STAT5 dependent: implications for the NOD STAT5B mutation in diabetes pathogenesis. Ann N Y Acad Sci 2006;1079:198-204 